Check for updates

Cite this: RSC Adv., 2017, 7, 34795

\title{
Perovskite photodetectors prepared by flash evaporation printing $\dagger$
}

\author{
Haoming Wei, ${ }^{\text {ab }} \mathrm{He} \mathrm{Ma},{ }^{c}$ Meiqian Tai, ${ }^{b}$ Yang Wei, (DD *a Dongqi Li, ${ }^{\text {a }}$ Xingyue Zhao, ${ }^{b}$ \\ Hong Lin, (D) *b Shoushan Fan ${ }^{\text {abd }}$ and Kaili Jiang*ad
}

Organometallic halide perovskites have expanded promising approaches for fabricating optoelectronic devices with low cost and high performance since their discovery. Here we propose a novel technique, flash evaporation printing, to prepare perovskite thin films with high quality in an efficient way, whose success is attributed to freestanding carbon nanotube films serving as the flash evaporator. It is convenient to print patterned perovskites using this method due to the compact deposition geometry. The patterned perovskite thin films were further manufactured to planar-type photodetectors, and the performance of the photoelectric devices was evaluated. The as-prepared photodetector shows a considerable peak responsivity and fast temporal response, proving flash evaporation printing an effective route for exploiting high-performance perovskite photodetectors, and this technique can find potential applications in more fields.

Received 10th April 2017

Accepted 7th July 2017

DOI: 10.1039/c7ra04061j

rsc.li/rsc-advances but rather dissociates into the free organic amine and hydrogen halide gas. ${ }^{17}$ Moreover, the conventional PVD method does not have the cost advantage due to the facility and the process.

In this study, we developed a flash evaporation printing (FEP) technique to prepare high-quality thin films of $\mathrm{CH}_{3} \mathrm{NH}_{3} \mathrm{PbI}_{3}$ $\left(\mathrm{MAPbI}_{3}\right)$ perovskite, which inherits the merits of PVD process and addresses its shortcomings as well. A cross-stacked superaligned carbon nanotube (CNT) film was employed as a flash evaporator for their ultrasmall heat capacity per unit area and fast thermal response ability. ${ }^{18-20} \mathrm{~A}$ flash evaporation transfer ribbon was then developed by coating $\mathrm{MAPbI}_{3}$ on the CNT evaporator. The transfer ribbon can be rapidly heated by sweeping laser beam on it, triggering a flash evaporation of $\mathrm{MAPbI}_{3}$. $\mathrm{A} \mathrm{MAPbI}_{3}$ perovskite thin film with high quality was successfully printed on the substrate by the gas phase transportation process from the hot CNTs to the cold substrate. Planar-type photodetectors were further fabricated by printing the perovskite thin films on Au interdigital electrodes. The photodetectors were found sensitive to a broadband response spectrum in visible light, showing a high responsivity $(R)$ of $4.98 \mathrm{~A} \mathrm{~W}^{-1}, 2.56 \mathrm{~A} \mathrm{~W}^{-1}$ at $460 \mathrm{~nm}$ and $694 \mathrm{~nm}$ under a bias voltage of $5 \mathrm{~V}$, respectively. Additionally, the as-prepared photodetectors showed a high response speed. The single evaporating source makes the FEP more simplified than the dual sources process, as it avoids the difficulties in accurate control of deposition rate and precursors ratio in customary vacuum evaporation. ${ }^{21}$ The FEP technique also simplifies the complicated thin film deposition facility into a compact printing module by using a CNT evaporator, which will have a significant cost advantage. The FEP technique has proven itself applicable to miscellaneous materials, and will be a promising candidate in printable electronics and flexible electronics. 
CNT films used in this work were dry spun from superaligned CNT arrays synthesized on silicon wafers by a lowpressure chemical vapor deposition (LPCVD) method. ${ }^{22,23} \mathrm{~A}$ cross-stacked CNT sheet with 15 single layers was coated onto a $25 \times 25 \mathrm{~mm}^{2}$ metal frame. After the stacking process, the CNT sheet was dipped in ethanol solvent and dried in air at room temperature to make CNTs firmly compact together, such a CNT sheet was the flash evaporator of the FEP technique. The perovskite precursor solution was consisted of $1 \mathrm{M} \mathrm{PbI}_{2}$ and $2.2 \mathrm{M} \mathrm{CH}_{3} \mathrm{NH}_{3} \mathrm{I}$ (MAI) in anhydrous solvent of dimethylformamide (DMF). Perovskite precursor solution of $100 \mu \mathrm{L}$ was then dropped and subsequently spin-coated on the CNT sheet at $2000 \mathrm{rpm}$ for 30 seconds. The transfer ribbon with $\mathrm{MAPbI}_{3}$ precursor was thus made after removing the solvents by annealing the composite film at $100{ }^{\circ} \mathrm{C}$ for 10 minutes. The scanning electron microscopy (SEM) image of the transfer ribbon is shown in the left panel of Fig. 1 inset.

The FEP technology is sketched in Fig. 1. The flash evaporation transfer ribbon with $\mathrm{MAPbI}_{3}$ was set on top of a substrate with a $1 \mathrm{~mm}$ gap, and the base pressure of the process chamber was $\sim 10^{-3}$ mbar. This compact geometry of the FEP simplifies the process chamber and is more cost effective than conventional evaporation facilities. The close distance between the source and the substrate can efficiently improve the material utilization during evaporation and thus effectively reduce lead pollution to the environment. As there is no significant change of the film quality when modulating the source-substrate distance from 1 to $3 \mathrm{~mm}$ (Fig. S1 $\dagger$ ), the $1 \mathrm{~mm}$ gap was employed to achieve a more compact structure. A scanning yttrium aluminum garnet (YAG) laser was introduced on the suspended transfer ribbon in the vacuum chamber through a quartz window. The wavelength of the laser was $1.06 \mu \mathrm{m}$, and the power output was optimized to be $12 \mathrm{~W}$. The laser spot, which was $\sim 1 \mathrm{~mm}$ in diameter, scanned on the CNT film along parallel lines with a pitch of $100 \mu \mathrm{m}$. The scanning speed was 1 $\mathrm{m} \mathrm{s}^{-1}$ in our experiments. The CNT evaporator can be heated to about $1100{ }^{\circ} \mathrm{C}$ instantaneously by the laser beam irradiation due to its ultrasmall heat capacity. The hot CNT film caused the

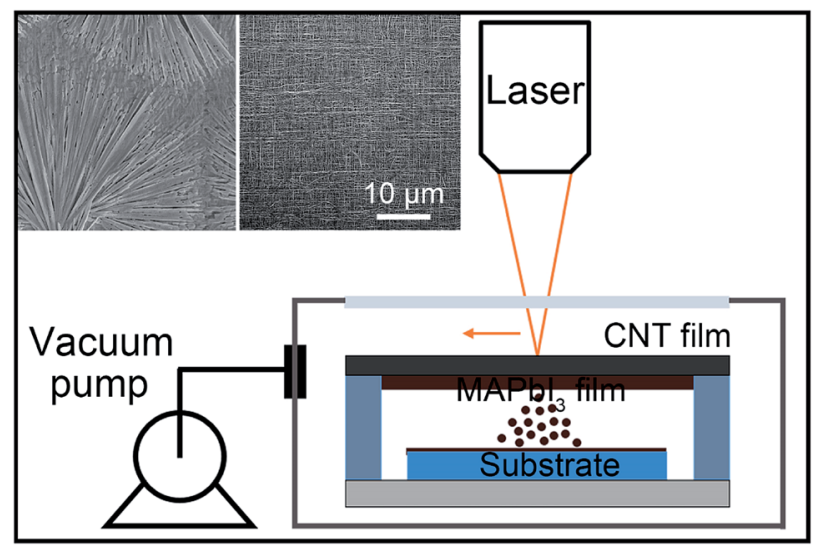

Fig. 1 Schematic depiction of the experimental setup of FEP. The inset are SEM images of the $\mathrm{MAPbl}_{3}$ transfer ribbon surface (left) and the clean CNT evaporator after flash evaporation (right). flash evaporation immediately and the perovskite precursor on the CNT evaporator is transfer printed onto the substrate via the vapour phase transportation process. It takes only about 4 seconds to print a $20 \times 20 \mathrm{~mm}^{2}$ perovskite film. The right panel of Fig. 1 inset shows the morphology of a transfer ribbon after flash evaporation, the clean surface without any residue suggests that the FEP is efficient.

The surface morphology of the as-printed perovskite film was investigated by SEM. The SEM image in Fig. 2a shows a typical morphology of the as-prepared perovskite thin film, indicating a high coverage. The grain size of the perovskite film was counted carefully for statistical analysis, and the crystallite size distribution is given in Fig. S2. $\uparrow$ The mean size of the 258 crystalline grains counted is $280 \mathrm{~nm}$ according to the statistics, and the sizes of most particles are over $200 \mathrm{~nm}$, accounting for more than $70 \%$. The crystalline structure was further studied by $\mathrm{X}$-ray diffraction (XRD) and the result is depicted in Fig. 2b. Intense diffraction peaks at $14.1^{\circ}, 28.4^{\circ}$, and $31.8^{\circ}$ of the curve could be assigned to (110), (220), and (310) planes of tetragonal $\mathrm{MAPbI}_{3}$ phase. ${ }^{\mathbf{4 , 1 3 , 1 4}}$ Moreover, the XRD pattern of $\mathrm{MAPbI}_{3}$ prepared using our FEP technique exhibits two extra peaks at $19.9^{\circ}$ and $24.4^{\circ}$, which were observed in the simulated perovskite XRD pattern reported by Graetzel et al. ${ }^{16}$ Besides, in the region of the (110) diffraction peak, there is barely signature of the diffraction peak for $\mathrm{PbI}_{2}$. All these analytical results indicate that a high level of film quality and phase purity can be acquired by the FEP methodology. In addition, the ultraviolet-visible (UVvis) spectroscopy was used to study the characteristic absorption of the perovskite films, which is given in Fig. 2c. It shows a band-to-band optical absorption edge at $\sim 800 \mathrm{~nm}(\sim 1.55 \mathrm{eV})$ for $\mathrm{MAPbI}_{3}$ perovskite, with an absorption plateau at wavelengths below $500 \mathrm{~nm} .^{6,24}$ The strong absorption of light indicates the perovskite film's possible photoelectric applications.

The FEP technique was further employed to fabricate planartype perovskite photodetectors. The fabrication procedures are illustrated in Fig. 3a. An array of interdigital gold electrodes was first deposited on the $\mathrm{SiO}_{2} / \mathrm{Si}$ wafer by conventional photolithography and e-beam evaporation process. The gap between $\mathrm{Au}$ electrodes was $20 \mu \mathrm{m}$. A perforated metal mask was then covered on the substrate and their patterns were aligned each other under an optical microscope. A central $500 \times 500 \mu \mathrm{m}^{2}$ square of each element was thus exposed for the perovskite thin film printing, as shown in the middle panel of Fig. 3a. After the FEP of perovskite thin film, an array of the planar-type photodetectors was successfully fabricated on the substrate. The effective illuminated area of $\mathrm{MAPbI}_{3}$ in each element was about $2.5 \times 10^{-7} \mathrm{~m}^{2}$. The optical micrographs in Fig. $3 \mathrm{~b}$ correspond to the three cartoons sketched in Fig. 3a, respectively. Moreover, the success in photodetector array fabrication also tells that the FEP technique is compatible with conventional semiconducting procedures, and it is also competent to print patterned thin films with a shadow mask strategy, which is essential for practical applications of a new printing methodology.

The photoelectric convertibility of the as-fabricated perovskite photodetectors was studied under the illumination via an optical microscope of a probe station. A $633 \mathrm{~nm}$ laser was first employed as the light source with the optical power densities 

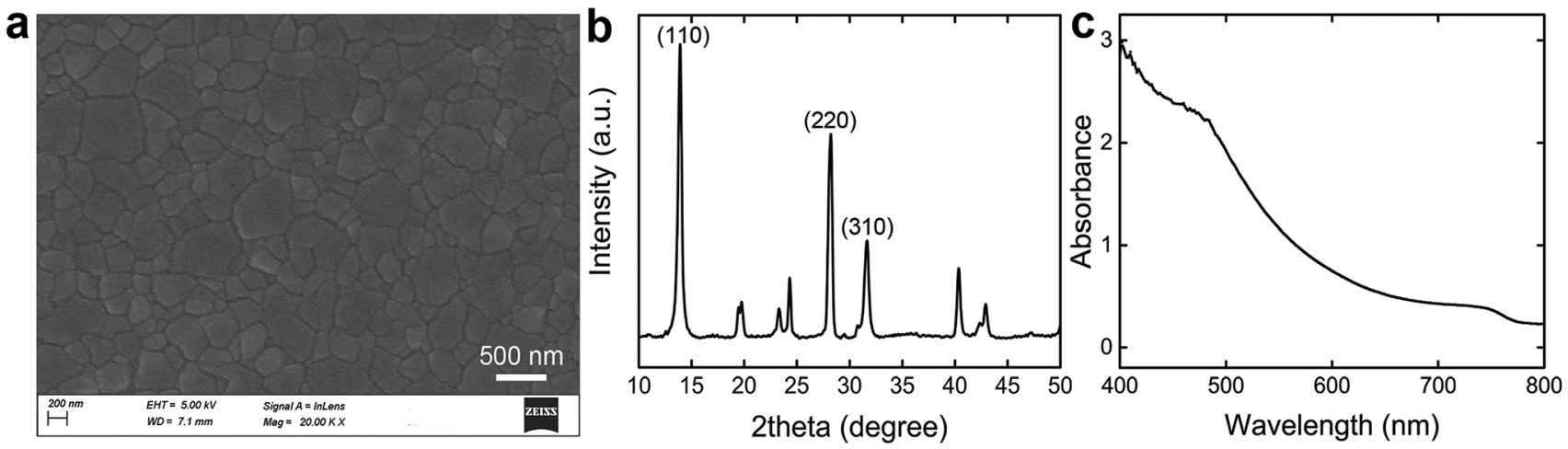

Fig. 2 Characterization of the as-printed thin films. (a) SEM image and (b) XRD pattern and (c) UV-vis absorption spectrum of the perovskite thin films deposited on FTO substrates.

varying from 4.79 to $32.99 \mathrm{~mW} \mathrm{~cm}{ }^{-2}$. The light intensities were measured with a calibrated power meter (Thorlabs PM100D). A bias was applied across the two pads of the device and $I-V$ curves under altered illumination power were plotted by Agilent B2902A sourcemeter. An applied voltage bias can provide the local electric field for the $\mathrm{MAPbI}_{3}$ perovskite film, leading to separate the photo-generated electrons and holes and decrease the electron-hole recombination rates, which is crucial for the high gain of the photodetector. After carriers dissociate, the electrons would migrate to the conduction band of $\mathrm{MAPbI}_{3}$ and further to the electrode with positive potential, while the holes would flow to the valence band and further to the electrode with negative potential. All measurements were performed in air at room temperature. The results are shown in Fig. 4a, in which the current density was defined as ratio of the current to the effective area of the device. The dark current was plotted as a reference (the black curve), which was as low as $0.75 \mathrm{nA}$ at $5 \mathrm{~V}$ bias. The dark resistance is about $6700 \mathrm{M} \Omega$. When the device was exposed to light, the photocurrent increased significantly. The $I-V$ curves show an almost linear and symmetrical behavior, indicating a well-symmetrical internal structure of the device, and the bright resistance at $32.99 \mathrm{~mW} \mathrm{~cm}^{-2}$ derived from the $I^{-}$ $V$ curves is $0.1 \mathrm{M} \Omega$. The $I-V$ characteristics of sandwiched cell consisting of fluorine-doped tin oxide (FTO)-coated glass/ $\mathrm{MAPbI}_{3}$ thin film/Au device were also measured, as shown in Fig. S3. $\dagger$ The DC (direct current) conductivity $\left(\sigma_{0}\right)$ can be determined from the slope of the $I-V$ plot, $I=\sigma_{0} A d^{-1} V,{ }^{25}$ where $A$ is area of sample $\left(0.26 \mathrm{~cm}^{2}\right)$ and $d$ is the thickness of the sample $(\sim 300 \mathrm{~nm})$. The determined conductivity was $0.0065 \mathrm{mS}$ $\mathrm{cm}^{-1}$.

The irradiance-dependent photocurrents at different bias were further plotted in Fig. 4b and fitted linearly, revealing a linear response across $\sim 4$ orders of intensity. The linear

a
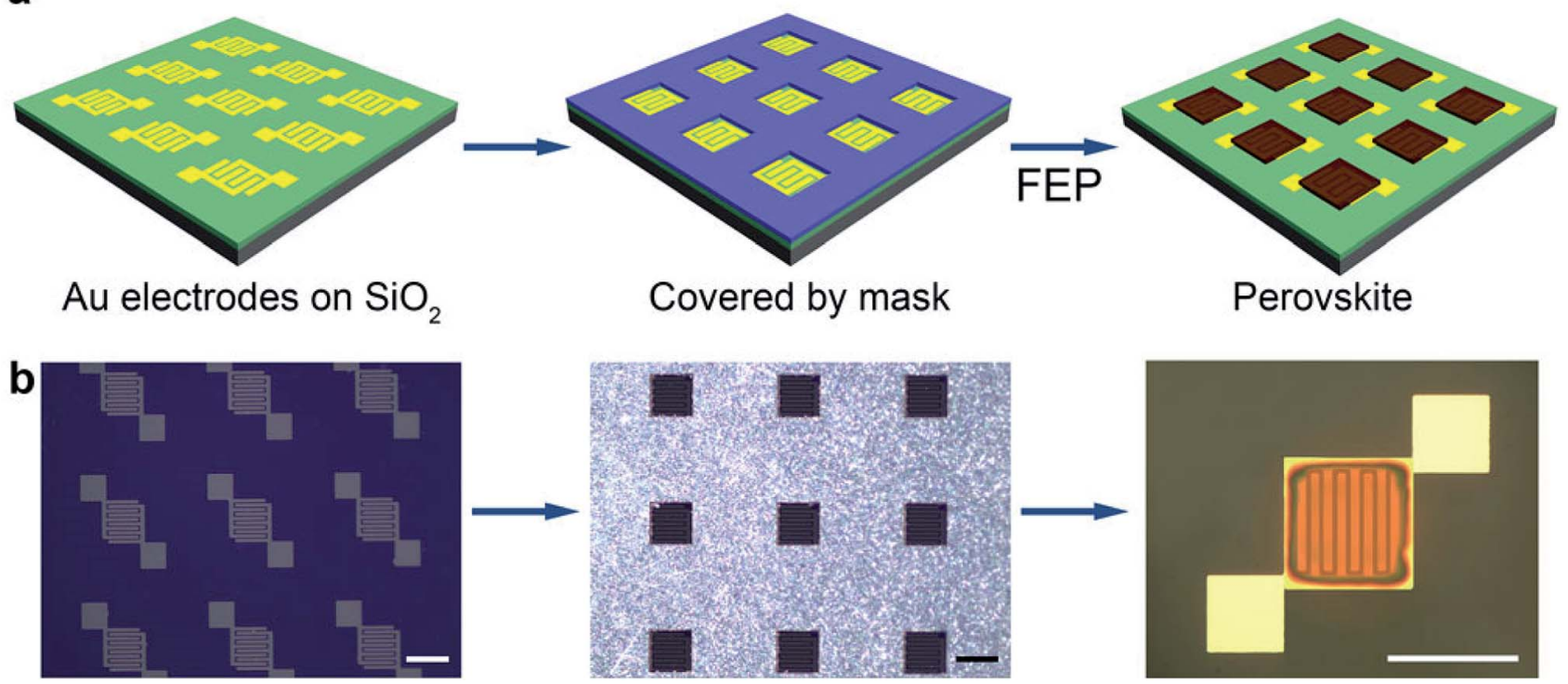

Fig. 3 (a) Schematic illustration of the as-fabricated perovskite photodetectors. (b) Optical micrographs of the gold interdigital fingers, the perforated metal mask and the as-fabricated photodetector element. The scale bar is $500 \mu \mathrm{m}$. 

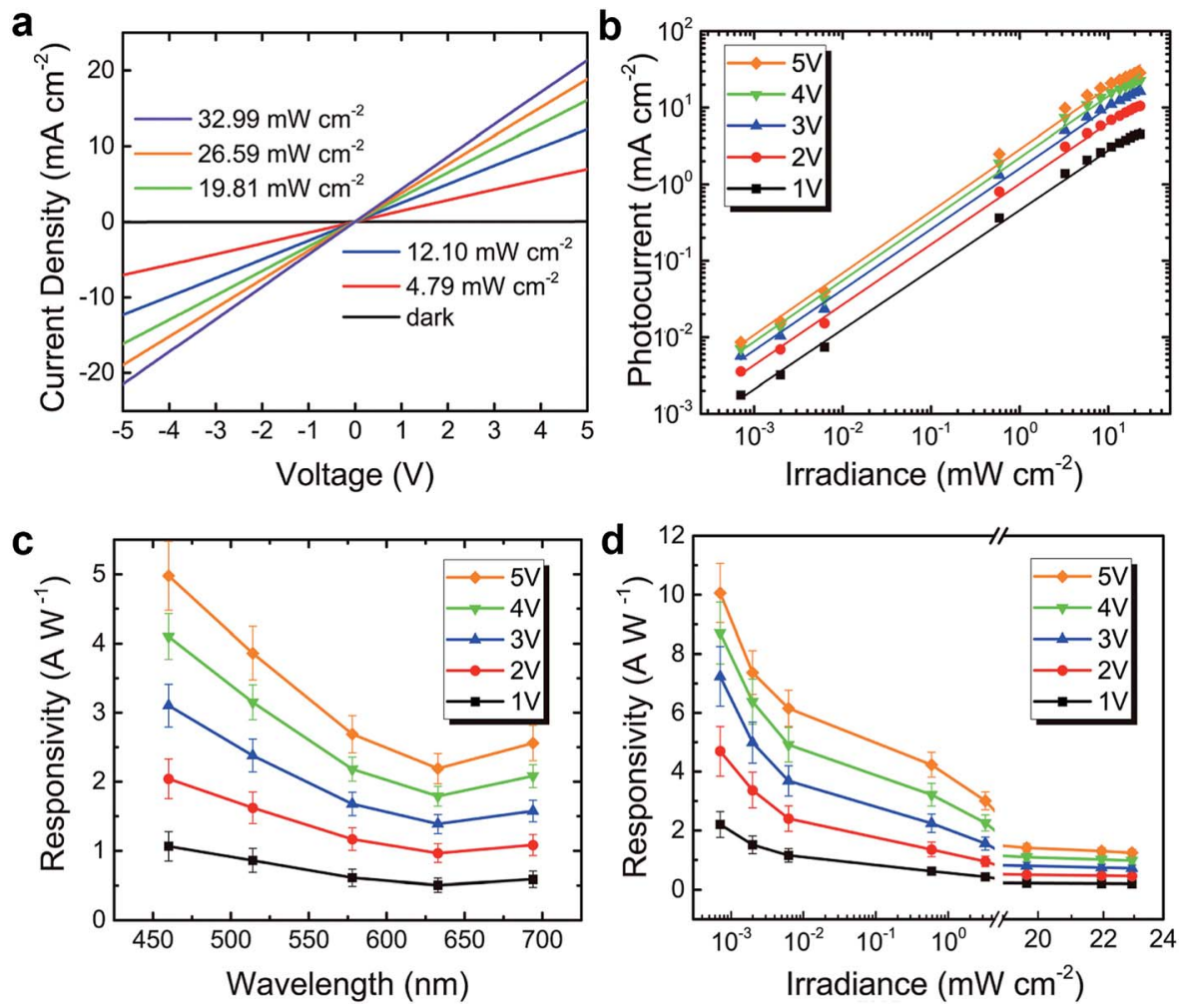

Fig. 4 (a) Photocurrent versus voltage curves under different irradiation densities. (b) Photocurrent linearity upon varying the $633 \mathrm{~nm}$ input irradiance at various bias. (c) Spectral responsivity of the as-fabricated device illuminated by bandpass-filtered light from Xe lamp. (d) Responsivity dependence on input irradiance at $633 \mathrm{~nm}$ with various bias.

dynamic range (LDR, typically quoted in $\mathrm{dB}$ ) can be calculated by the equation:

$$
\mathrm{LDR}=20 \log \frac{J_{\mathrm{ph}}^{*}}{J_{\mathrm{dark}}}
$$

where $J_{\mathrm{ph}}^{*}$ is the photocurrent measured at a light intensity of 1 $\mathrm{mW} \mathrm{cm}{ }^{-2} \cdot{ }^{26}$ The calculated linear dynamic range (LDR) is $\sim 80$ $\mathrm{dB}$, which is higher than InGaAs photodetectors (66 dB).

Responsivity $(R)$, the ratio of photocurrent to the incident light intensity, can be expressed by:

$$
R=\frac{J_{\mathrm{ph}}}{L_{\text {light }}}
$$

where $J_{\mathrm{ph}}$ is the photocurrent and $L_{\text {light }}$ is the incident light intensity. Since $R$ indicates how efficiently the photodetector responds to an optical signal, it is another crucial figure of merit for photodetectors. To measure the responsivity, the photodetector was illuminated by monochromatic light which was derived from a xenon lamp with bandpass filters. The responsivities under 5 different wavelengths were thus studied, the wavelengths and the corresponding illumination power densities are $460 \mathrm{~nm} 0.04 \mathrm{~mW} \mathrm{~cm}^{-2}, 514 \mathrm{~nm} 0.13 \mathrm{~mW} \mathrm{~cm}^{-2}, 578 \mathrm{~nm}$ $0.33 \mathrm{~mW} \mathrm{~cm}{ }^{-2}, 633 \mathrm{~nm} 0.38 \mathrm{~mW} \mathrm{~cm}^{-2}$ and $694 \mathrm{~nm} 0.08 \mathrm{~mW}$ $\mathrm{cm}^{-2}$, respectively. The device displays a photoresponse under these wavelengths, as shown in Fig. 4c. The responsivity is $4.98 \mathrm{~A} \mathrm{~W}^{-1}$ at $460 \mathrm{~nm}$ wavelength with a $5 \mathrm{~V}$ bias voltage, and it can also reach around $1 \mathrm{~A} \mathrm{~W}^{-1}$ even at a relatively low bias voltage of $1 \mathrm{~V}$. This performance is comparable to commercial large-area silicon photodiodes detecting at the same wavelength, ${ }^{27}$ due to the strong direct-bandgap absorption of perovskite films. It should be noted that the characterization of responsivity below $460 \mathrm{~nm}$ and over $694 \mathrm{~nm}$ is not successful because of the limitation of the bandpass filters used, otherwise it is expectable that the photodetector will have a wide-range photoresponse from ultraviolet to visible light, considering the absorption characteristics of $\mathrm{MAPbI}_{3}$. Furthermore, the irradiance power-dependent responsivity was evaluated at varying bias with the $633 \mathrm{~nm}$ laser, as shown in Fig. $4 \mathrm{~d}$. It can be found that the responsivity decreases gradually as the irradiance increases. The lower responsivity at higher powers should be attributed to the reduced photoconductive gain by the dominated shallow trap states with shorter lifetime compared with deep traps states, ${ }^{28-30}$ thus the highest $R$ value can be measured at the lowest detectable irradiance power intensity. Under $0.71 \mu \mathrm{W} \mathrm{cm}{ }^{-2}$ irradiance, the measured $R$ values presented in Fig. $4 \mathrm{~d}$ are $10.06 \mathrm{~A} \mathrm{~W}^{-1}$ at $5 \mathrm{~V}$ and $2.20 \mathrm{~A} \mathrm{~W}^{-1}$ at $1 \mathrm{~V}$, respectively. All these responsivity measurements demonstrated that the as-prepared photodetectors behaved well.

To further investigate the temporal response of the photodetector, a supercontinuum white light source was employed as a modulated illumination..$^{31}$ The pulse duration of the light source was less than $2 \mathrm{~ns}$ and the frequency was set at $5000 \mathrm{~Hz}$. The transient photocurrent response of the device was recorded by an oscilloscope and shown in Fig. 5a. One of the transient 

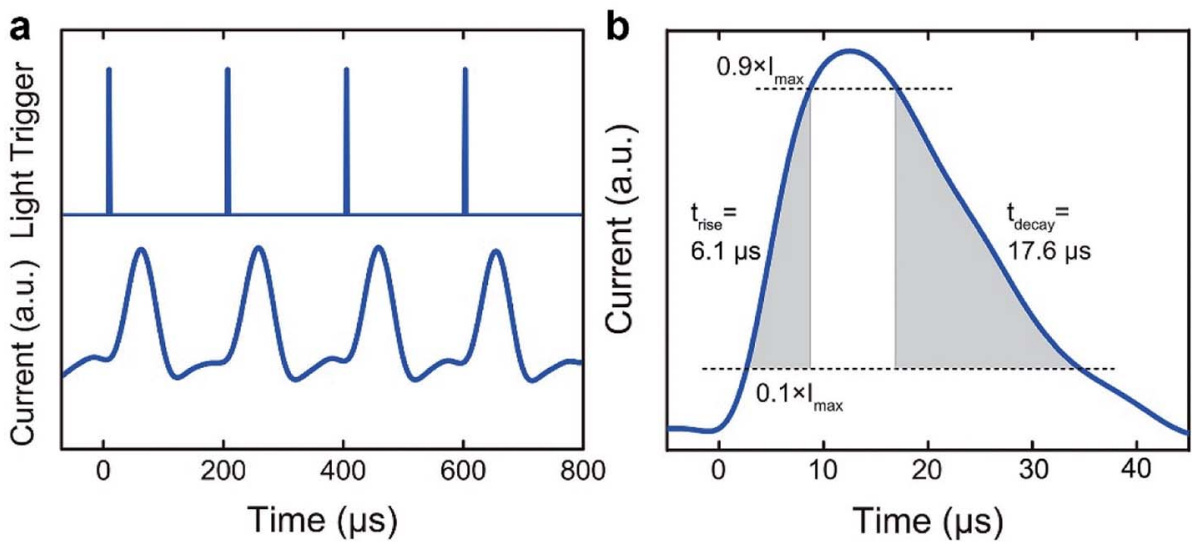

Fig. 5 Perovskite photodetector speed at zero bias. (a) Transient current output of the device to a time-varying incident light signal. (b) Photocurrent rise and decay.

signals was presented in Fig. 5b to further characterize the rise time and fall time. The rise time was defined as the time to transition between $10 \%$ of the maximum photocurrent to $90 \%$ of the maximum photocurrent; the fall time was from $90 \%$ down to $10 \%$. The rise time and the fall time of the photodetector were around 6.1 and $17.6 \mu$ s, respectively. The decay rate is slower than the rise rate, which can be typically attributed to the diffusive contribution to photocurrent during discharging. ${ }^{32}$ The temporal response presented here are faster than some organic, quantum dot and hybrid photodetectors in the literature ${ }^{33-38}$ which can naturally extend the scope of device applications and reveal the great potentials of the as-developed FEP methodology.

\section{Conclusions}

To conclude, by utilizing superaligned CNT films with ultrasmall heat capacity and fast thermal response as the flash evaporator, a convenient FEP technology was developed and used to print perovskite thin films with high quality. The as-prepared films' property and morphology were proved favorable by XRD and SEM. As the FEP technology offers a simple but efficient solution to print patterned thin films, it is employed to prepare perovskite patterns using a shadow mask, and the patterning printing can also be realized by a point-by-point printing thanks to its compact geometry. The patterned perovskite thin films were further fabricated into planar-type photodetectors, and the photoresponse performance of the devices were evaluated. The lowest detectable irradiance power density was evaluated to be $0.71 \mu \mathrm{W}$ $\mathrm{cm}^{-2}$, under which the highest $R$ values of $10.06 \mathrm{~A} \mathrm{~W}^{-1}$ at $5 \mathrm{~V}$ and $2.20 \mathrm{~A} \mathrm{~W}^{-1}$ at $1 \mathrm{~V}$ were achieved, respectively. The transient photoresponse measurement showed the device had a very fast photoresponse speed, the rise time was $6.1 \mu$ s and the fall time was $17.6 \mu \mathrm{s}$, faster than some organic, quantum dot and hybrid photodetectors according to the literature. In addition, further enhancements of the photodetector performance may be predictable by improving the interface between the perovskite films and gold electrodes. These results demonstrate that FEP is an effective way for exploiting high-performance perovskite photodetectors, and FEP is also a promising methodology for printing other functional materials, finding its prospective applications in printed electronics, organic electronics and future flexible electronics.

\section{Acknowledgements}

This work was financially supported by the National Basic Research Program of China (No. 2012CB932301), and the National Natural Science Foundation of China (No. 51472142, 51102147).

\section{Notes and references}

1 M. M. Lee, J. Teuscher, T. Miyasaka, T. N. Murakami and H. J. Snaith, Science, 2012, 338, 643-647.

2 M. A. Loi and J. C. Hummelen, Nat. Mater., 2013, 12, 10871089.

3 G. Hodes, Science, 2013, 342, 317-318.

4 J. Y. Jeng, Y. F. Chiang, M. H. Lee, S. R. Peng, T. F. Guo, P. Chen and T. C. Wen, Adv. Mater., 2013, 25, 3727-3732.

5 C. C. Stoumpos, C. D. Malliakas and M. G. Kanatzidis, Inorg. Chem., 2013, 52, 9019-9038.

6 S. D. Stranks, G. E. Eperon, G. Grancini, C. Menelaou, M. J. P. Alcocer, T. Leijtens, L. M. Herz, A. Petrozza and H. J. Snaith, Science, 2013, 342, 341-344.

7 G. Xing, N. Mathews, S. Sun, S. S. Lim, Y. M. Lam, M. Grätzel, S. Mhaisalkar and T. C. Sum, Science, 2013, 342, 344-347.

8 P. Gao, M. Grätzel and M. K. Nazeeruddin, Energy Environ. Sci., 2014, 7, 2448-2463.

9 G. Xing, N. Mathews, S. S. Lim, N. Yantara, X. Liu, D. Sabba, M. Grätzel, S. Mhaisalkar and T. C. Sum, Nat. Mater., 2014, 13, 476-480.

10 H. Zhou, Q. Chen, G. Li, S. Luo, T. B. Song, H. S. Duan, Z. Hong, J. You, Y. Liu and Y. Yang, Science, 2014, 345, 542-546.

11 H. S. Kim, C. R. Lee, J. H. Im, K. B. Lee, T. Moehl, A. Marchioro, S. J. Moon, R. Humphry-Baker, J. O. Yum, J. E. Moser, M. Grätzel and N. G. Park, Sci. Rep., 2012, 2, 591. 
12 J. H. Heo, S. H. Im, J. H. Noh, T. N. Mandal, C. S. Lim, J. A. Chang, Y. H. Lee, H. J. Kim, A. Sarkar, M. K. Nazeeruddin, M. Grätzel and S. I. Seok, Nat. Photonics, 2013, 7, 486-491.

13 J. Burschka, N. Pellet, S. J. Moon, R. Humphry-Baker, P. Gao, M. K. Nazeeruddin and M. Grätzel, Nature, 2013, 499, 316319.

14 M. Liu, M. B. Johnston and H. J. Snaith, Nature, 2013, 501, 395-398.

15 Q. Chen, H. Zhou, Z. Hong, S. Luo, H. S. Duan, H. H. Wang, Y. Liu, G. Li and Y. Yang, J. Am. Chem. Soc., 2014, 136, 622625.

16 O. Malinkiewicz, A. Yella, Y. H. Lee, G. M. Espallargas, M. Graetzel, M. K. Nazeeruddin and H. J. Bolink, Nat. Photonics, 2014, 8, 128-132.

17 D. B. Mitzi, M. T. Prikas and K. Chondroudis, Chem. Mater., 1999, 11, 542-544.

18 K. Liang, D. B. Mitzi and M. T. Prikas, Chem. Mater., 1998, 10, 403-411.

19 L. Xiao, Z. Chen, C. Feng, L. Liu, Z. Bai, Y. Wang, L. Qian, Y. Zhang, Q. Li, K. Jiang and S. Fan, Nano Lett., 2008, 8, 4539-4545.

20 Y. Wei, X. Lin, K. Jiang, P. Liu, Q. Li and S. Fan, Nano Lett., 2013, 13, 4795-4801.

21 H. Wei, Y. Wei, X. Lin, P. Liu, S. Fan and K. Jiang, Nano Lett., 2015, 15, 1843-1848.

22 K. Jiang, Q. Li and S. Fan, Nature, 2002, 419, 801.

23 K. Jiang, J. Wang, Q. Li, L. Liu, C. Liu and S. Fan, Adv. Mater., 2011, 23, 1154-1161.

24 Z. Xiao, C. Bi, Y. Shao, Q. Dong, Q. Wang, Y. Yuan, C. Wang, Y. Gao and J. Huang, Energy Environ. Sci., 2014, 7, 2619-2623.

25 J. H. Heo, H. J. Han, D. Kim, T. K. Ahn and S. H. Im, Energy Environ. Sci., 2015, 8, 1602-1608.
26 X. Gong, M. Tong, Y. Xia, W. Cai, J. S. Moon, Y. Cao, G. Yu, C. L. Shieh, B. Nilsson and A. J. Heeger, Science, 2009, 325, 1665-1667.

27 O. Bisi, S. Campisano and L. Pavesi, Silicon-Based Microphotonics: From Basics to Applications, IOS Press, Bologna, 1999.

28 I. Ka, V. Le Borgne, K. Fujisawa, T. Hayashi, Y. A. Kim, M. Endo, D. Ma and M. A. El Khakani, Sci. Rep., 2016, 6, 20083.

29 G. Konstantatos, I. Howard, A. Fischer, S. Hoogland, J. Clifford, E. Klem, L. Levina and E. H. Sargent, Nature, 2006, 442, 180-183.

30 G. Konstantatos, J. Clifford, L. Levina and E. H. Sargent, Nat. Photonics, 2007, 1, 531-534.

31 W. Wu, J. Yue, X. Lin, D. Li, F. Zhu, X. Yin, J. Zhu, J. Wang, J. Zhang, Y. Chen, X. Wang, T. Li, Y. He, X. Dai, P. Liu, Y. Wei, J. Wang, W. Zhang, Y. Huang, L. Fan, L. Zhang, Q. Li, S. Fan and K. Jiang, Nano Res., 2015, 8, 2721-2732.

32 A. Rogalski, Infrared Detectors, CRC Press, 2nd edn, 2010.

33 X. Hu, X. Zhang, L. Liang, J. Bao, S. Li, W. Yang and Y. Xie, Adv. Funct. Mater., 2014, 24, 7373-7380.

34 Z. Lian, Q. Yan, Q. Lv, Y. Wang, L. Liu, L. Zhang, S. Pan, Q. Li, L. Wang and J. Sun, Sci. Rep., 2015, 5, 16563.

35 Y. Jin, J. Wang, B. Sun, J. C. Blakesley and N. C. Greenham, Nano Lett., 2008, 8, 1649-1653.

36 V. Sukhovatkin, S. Hinds, L. Brzozowski and E. H. Sargent, Science, 2009, 324, 1542-1544.

37 F. Guo, B. Yang, Y. Yuan, Z. Xiao, Q. Dong, Y. Bi and J. Huang, Nat. Nanotechnol., 2012, 7, 798-802.

38 H. Y. Chen, K. F. LoMichael, G. Yang, H. G. Monbouquette and Y. Yang, Nat. Nanotechnol., 2008, 3, 543-547. 